\section{Utilização do e-SUS AB e fatores associados ao registro de procedimentos e consultas da atenção básica nos municípios brasileiros}

\author{
e-SUS AB use and factors associated with \\ recording primary care procedures and \\ consultations in Brazilian municipalities
}

\section{Utilización del e-SUS AB y factores asociados con el registro de procedimientos y consultas de la atención básica en municipios brasileños}

\section{Resumo}

O objetivo foi avaliar o impacto do e-SUS AB na notificação de procedimentos e consultas no Sistema de Informação Ambulatorial do Sistema Único de Saúde (SIA/SUS) dos municípios brasileiros. Estudo ecológico com comparação longitudinal antes, durante e depois do período de implantação do e-SUS $A B$ (junho de 2013 a maio de 2015) e, durante a implantação, comparação seccional entre municípios em fase implantado, intermediário e incipiente. As taxas foram descritas por médias e desvio padrão, e analisadas por regressão binomial negativa. Os municípios com e-SUS AB implantado em novembro 2014 eram de pequeno porte, com menor Índice de Desenvolvimento Humano Municipal (IDH-M), menor renda per capita, maiores taxas de enfermeiros, alta cobertura de Estratégia Saúde da Família - ESF (> 80\%). Não foram observadas diferenças nas taxas de procedimentos ou consultas entre municípios de acordo com a fase de implantação e não houve aumento ao longo do tempo. Municípios com cobertura de $100 \%$ da ESF apresentam maiores taxas de procedimentos (razão de taxas [RT] = 1,44; IC95\%: 1,29-1,62) e de consultas $(R T=1,26$; IC95\%: 1,15-1,39); municípios com mais de 100 mil habitantes registraram menores taxas de procedimentos $(R T=0,68 ; I C 95 \%: 0,57-0,81) e$ de consultas $(R T=0,54$; IC95\%: 0,47-0,63). Houve menores taxas de procedimentos em todas as regiões quando comparadas com a Região Norte, tendo a Nordeste RT de 0,34 (IC95\%: 0,30-0,40) vez menor e a Região Sul, RT de 0,35 (IC95\%: 0,30-0,41) menor que a Região Norte. Outras análises quanto ao uso do sistema e suas implicações nos processos de trabalho das equipes devem ser aprofundadas para apoiar os gestores no uso e manutenção do sistema.

Registros Eletrônicos de Saúde; Sistemas de Informação em Saúde;

Estratégia Saúde da Família; Atenção Primária à Saúde
Moara Ailane Thum 1 Julio Baldisserotto 1,2 Roger Keller Celeste 2

doi: 10.1590/0102-311X00029418

\section{Correspondência}

R. K. Celeste

Departamento de Odontologia Preventiva e Social, Faculdade de Odontologia, Universidade Federal do Rio Grande do Sul. Rua Ramiro Barcelos 2492, Porto Alegre, RS 90035-003, Brasil. roger.keller@ufrgs.br

1 Programa de Pós-graduação em Avaliação de Tecnologias para o Sistema Único de Saúde, Grupo Hospitalar Conceição, Porto Alegre, Brasil.

2 Faculdade de Odontologia, Universidade Federal do Rio Grande do Sul, Porto Alegre, Brasil. 


\section{Introdução}

A coleta de informações na atenção básica vinha sendo realizada por meio do Sistema de Informação da Atenção Básica (SIAB) desde 1994, com a adoção da Estratégia Saúde da Família (ESF) como modelo de reorganização da atenção da básica. Desde 2013, o Ministério da Saúde vem implementando o novo Sistema de Informação em Saúde para a Atenção Básica (SISAB) por intermédio da estratégia "e-SUS AB". Essa ferramenta tecnológica é executada usando-se um software que funciona como Coleta de Dados Simplificada (CDS) ou Prontuário Eletrônico do Cidadão (PEC). A escolha do modelo utilizado depende da especificidade e disponibilidade dos recursos de informática de cada local 1. O PEC, além de funcionar como prontuário médico para registro do atendimento individual de cada paciente, também organiza a demanda da unidade e gerencia a agenda dos profissionais, assim como notifica procedimentos realizados ao Sistema de Informação Ambulatorial do Sistema Único de Saúde (SIA/SUS). Os altos custos de implantação de um sistema informatizado nas mais de $30 \mathrm{mil}$ unidades básicas de saúde do país (Ministério da Saúde. Unidades Básicas de Saúde - UBS - conjuntos de dados. http://dados.gov.br/dataset/unidades-basicas-de-saude-ubs, acessado em 18/Out/2018), os resultados esperados de qualificação dos registros, a diminuição do tempo gasto com burocracia e a racionalização dos recursos ainda carecem de monitoramento e avaliação, dada a recente utilização do sistema. Algumas avaliações ainda localizadas de implantação do e-SUS AB e de relatos de experiências têm sido publicadas 2 , no entanto, não há, no nosso conhecimento, uma avaliação de abrangência nacional do e-SUS AB em território brasileiro.

Os sistemas de informação em saúde, aliados às novas tecnologias de informação e comunicação (TIC) por meio dos registros eletrônicos em saúde, produzem de forma organizada informações sobre a eficiência das ações e situações de saúde da população ${ }^{3}$. Os sistemas informatizados permitem o registro integrado dos dados dos pacientes e que os profissionais atuem com as melhores práticas disponíveis visando à qualidade do cuidado 4,5. No entanto, estudos têm apontado que, mesmo em países desenvolvidos, um conjunto multivariado de fatores interfere na incorporação e implementação de tecnologias de informação em saúde 6,7. Dentre esses fatores estão a qualidade da interface com o usuário, funcionalidade dos recursos e usabilidadade, qualidade dos dados coletados e integração com outros sistemas ${ }^{6}$. No entanto, a inclusão ou implementação de uma nova tecnologia por si só não garante melhorias na atenção à saúde. Uma revisão sistemática 7 sobre adoção de registro eletrônico na atenção primária, concluiu que a qualidade do cuidado, a segurança do paciente, a relação entre paciente/profissional e os serviços não foram afetados pela implantação dos sistemas de informação. A revisão revelou ainda que fatores técnicos dos sistemas de informação podem interagir com as características sociais (processo de trabalho, condições físicas, demandas) em que se inserem os serviços de saúde, podendo dificultar este processo de implantação de uma nova tecnologia. A perspectiva sociotécnica considera como as características técnicas de um sistema de informação em saúde interagem com as condições sociais onde se encontra o serviço de saúde 7 . Assim, as condições sociais em que vive a população, a organização dos sistemas locais de saúde e a diversidade regional devem ser consideradas na perspectiva da avaliação do impacto da introdução das tecnologias de informação sobre a atenção à saúde da população. Dados do Programa Nacional de Melhoria do Acesso e da Qualidade da Atenção Básica (PMAQ) 8 encontraram que existe associação entre a incorporação de tecnologias de informação e a qualidade da atenção e, portanto, cabe aos gestores o desenvolvimento e a implementação de políticas que possam acelerar o processo de incorporação de TIC no Brasil, contribuindo para a melhoria do cuidado prestado pelas equipes de atenção básica 9 .

Pode-se esperar que esse processo de reorganização da coleta de informações por meio do SISAB e da informatização do SIA/SUS qualifique os dados registrados pelas equipes 1. Essa configuração torna o SISAB o sistema centralizador dos dados transmitidos pelos municípios, mesmo os que utilizam outros sistemas eletrônicos, privados ou não. Além de uma qualificação da informação, o sistema deveria facilitar o registro e, no caso do PEC, quem executa o registro faz a notificação pessoal, sendo talvez capaz de reduzir subnotificação. Há estudos que apontaram um desempenho moderado no quantitativo de consultas médicas na produção ambulatorial do SUS, abaixo do preconizado pelo Ministério da Saúde 10,11. Há que se considerar também que a enfermagem apresenta um novo papel na organização dos processos de trabalho nas unidades de saúde, ficando cada vez mais distante das práticas assistenciais 12 , que poderiam resultar em mais atendimentos. 
Dada a recente utilização da estratégia e-SUS AB como novo sistema de informação agregado à informatização das equipes, faz-se necessário avaliar as implicações que podem ocorrer no registro dos dados para o sistema de saúde no país. Sendo assim, o objetivo deste trabalho foi avaliar o impacto da implantação do e-SUS AB na notificação dos procedimentos e de consultas por intermédio do SIA/SUS pelos municípios brasileiros.

\section{Metodologia}

Trata-se de um estudo ecológico com duas linhas de análise. A primeira consiste numa comparação temporal, em nível nacional, em três momentos: (1) antes (junho de 2011 a maio de 2013); (2) durante (junho de 2013 a maio de 2015) e (3) depois (junho de 2015 a maio de 2016) do processo de implantação do e-SUS AB nos procedimentos e consultas registrados no SIA/SUS. O período representa um recorte de dois anos antes da implantação do e-SUS AB, sua implantação e um ano após a implantação do sistema.

A outra linha de análise consiste em uma comparação de forma seccional no período "2", mencionado no parágrafo anterior, entre municípios com e-SUS AB em três níveis de implantação: (1) implantado (mais de 60\% das unidades utilizando o sistema); (2) em processo intermediário de implantação (30\% a 60\% dos serviços enviando dados) e (3) não iniciado ou incipiente (nenhuma ou ao menos uma unidade de saúde iniciou a implantação do e-SUS AB). Tal classificação foi adotada pelo Departamento de Atenção Básica do Ministério da Saúde e é definida por critérios de envio de dados ao SIA/SUS. Os períodos foram escolhidos devido à oficialização e obrigatoriedade do uso do e-SUS $\mathrm{AB}$ a partir da competência de junho de 2015 13, quanto o percentual de municípios com o sistema alcançou virtualmente $100 \%$. Quanto à implantação, o período de corte foi definido em novembro de 2014, quando um percentual de 46,5\% dos municípios já alimentava o SIA/SUS por meio do e-SUS $\mathrm{AB}$ em pelo menos uma unidade de saúde. Para a análise foi utilizada a média dos meses de outubro, novembro e dezembro de 2014.

\section{Fonte de dados}

Os dados foram extraídos de bancos de dados secundários de livre acesso, disponibilizados pelo Ministério da Saúde e outros órgãos federais, dentre eles: SIA/SUS, Cadastro Nacional de Estabelecimentos de Saúde (CNES), Instituto Brasileiro de Geografia e Estatística (IBGE) e Atlas de Desenvolvimento Humano do Brasil (ATLAS). A coleta de dados foi realizada em janeiro de 2017 pelos pesquisadores. Todas as informações foram extraídas em nível municipal e os bancos foram unidos utilizando-se os códigos do IBGE para identificação dos municípios.

\section{Procedimento amostral}

Foram usados os dados de todos os municípios brasileiros que estavam alimentando com regularidade o SIA/SUS. Os procedimentos foram eleitos de acordo com a tabela SIGTAP de procedimentos do SUS (Sistema de Gerenciamento da Tabela Unificada de Procedimentos do SUS. http:// sigtap.datasus.gov.br/tabela-unificada/app/sec/inicio.jsp), sendo excluídos os de média e alta complexidades.

Em consonância com o Departamento de Informática do SUS (DATASUS), a forma de organização "consultas médicas/outros profissionais de nível superior" foi nomeada como "consultas" e engloba 22 códigos de notificação, dos quais dez são de competência da atenção básica. São eles: Consulta ao Paciente Curado de Tuberculose (tratamento supervisionado) (03.01.01.001-3), Consulta com Identificação de Casos Novos de Tuberculose (03.01.01.002-1), Consulta de Profissionais de Nível Superior na Atenção Básica (exceto médico) (03.01.01.003-0), Consulta Médica em Atenção Básica (03.01.01.006-4), Consulta para Acompanhamento de Crescimento e Desenvolvimento (puericultura) (03.01.01.008-0), Consulta para Avaliação Clínica do Fumante (03.01.01.009-9), Consulta Pré-Natal (03.01.01.011-0), Consulta Puerperal (03.01.01.012-9), Consulta/Atendimento Domiciliar (03.01.01.013-7), Primeira Consulta Odontológica Programática (03.01.01.015-3). 
Já o grupo "procedimentos”, categorizado como "atendimentos de enfermagem” no DATASUS, contém 17 procedimentos. Nele foram avaliadas 11 ações rotineiramente notificadas pela atenção básica: Lavagem Gástrica (03.01.10.012-8), Ordenha Mamária (03.01.10.013-6), Oxigenoterapia (03.01.10.014-4), Retirada de Pontos de Cirurgias Básicas (por paciente) (03.01.10.015-2), Sondagem Gástrica(03.01.10.017-9), Terapia de Reidratarão Oral(03.01.10.018-7), Administração de Medicamentos em Atenção Básica (por paciente) (03.01.10.002-0), Aferição de Pressão Arterial (03.01.10.003-9), Cateterismo Vesical de Alívio (03.01.10.004-7), Cateterismo Vesical de Demora (03.01.10.005-5), Inalação/Nebulização (03.01.10.010-1).

As taxas municipais foram construídas tendo como numerador as médias mensais de procedimentos/consultas e como denominador o número de habitantes. Vários códigos de procedimentos/ consultas foram agrupados (ver os dois parágrafos anteriores) por um período de aproximadamente dois anos antes (junho de 2011 a maio de 2013), dois anos durante (junho de 2013 a maio de 2015) e um ano depois (junho de 2015 a maio de 2016) da implantação. Nas análises transversais, a média mensal equivale ao período de três meses (outubro-dezembro de 2014). Dessa forma, foram minimizados possíveis erros de notificação e eventuais flutuações aleatórias que podem acontecer para procedimentos específicos raros com pequenos denominadores.

\section{Variáveis de interesse e categorização}

Foram utilizadas como covariáveis: o Índice de Desenvolvimento Humano Municipal (IDH-M), o Produto Interno Bruto (PIB) per capita, a macrorregião, o porte municipal, o percentual de população urbana, a taxa de médicos e de enfermeiros e a taxa de unidades de saúde de atenção básica. Algumas informações de perfil demográfico também foram usadas: proporção de idosos ( 70 anos), proporção de crianças (< 10 anos), proporção de mulheres, proporção da população com planos privados de saúde (ambulatoriais e hospitalares) e proporção de pessoas cobertas pela ESF.

\section{Análise estatística}

A descrição das taxas de procedimentos e consultas nos municípios brasileiros foi feita em médias e desvio padrão de acordo com o nível de implantação e comparações testadas com testes não paramétricos apropriados. O efeito da adesão ao sistema e-SUS AB foi avaliado utilizando-se taxas do período em que metade dos municípios tinha > 60\% das suas unidades de saúde com o sistema implantado, o que ocorreu em novembro de 2014. Tal associação foi calculada ajustando seu efeito em modelos de regressão múltipla. Utilizou-se regressão binomial negativa múltipla tendo como desfecho o número de procedimentos informados e como denominador o número de habitantes no município. Análises preliminares mostraram sobredispersão da variância do desfecho de 2,2 vezes o esperado (Likelihood-ratio test of alpha $=0, \mathrm{p}<0,01$ ) para uma distribuição de Poisson. Os coeficientes exponenciados podem ser interpretados como razões de taxas. O critério de seleção de variáveis incluiu todas as variáveis que poderiam afetar o desfecho. Além disso, testou-se a interação entre a principal variável de interesse com a variável macrorregião, em virtude de diferenças regionais. A remoção de variáveis do modelo e definição do modelo final foram baseadas em indicadores de ajuste: Akaike Information Criterion (AIC) e qualidade de resíduos. Utilizou-se o mesmo modelo de variáveis para ambos os desfechos: procedimentos e consultas. Todas as análises foram feitas usando-se o software Stata 13.1 (https://www.stata.com).

\section{Resultados}

Foram utilizados dados de todos os municípios brasileiros $(\mathrm{n}=5.564)$ que, de acordo com o Censo Demográfico de 2010 (IBGE. http://www.ibge.gov.br), tem uma proporção média de urbanização de $63,83 \%$. Devido a dados faltantes em diferentes fontes de dados e alterações no número de municípios durante os anos da pesquisa, o número total de municípios varia, sendo o menor valor para o modelo final de 5.440, representando 2,2\% de perdas. A média do PIB per capita entre 2011 e 2013 foi de R\$ 15.861,38, sendo o mínimo R\$ 3.047,03 e o máximo R\$ 704.340,00. Os procedimentos e consultas 
estudados foram selecionados com base no envio dos dados ao Ministério da Saúde pelos municípios, não havendo nenhuma exclusão. Verificaram-se as taxas médias de 0,48 unidade de saúde, de 0,60 enfermeiro e de 1,05 médico por mil habitantes/mês em todos os municípios (dados não disponibilizados em tabela).

O perfil dos municípios que tinham o e-SUS AB implantado no momento intermediário (novembro de 2014) mostrou que a maioria era de pequeno porte, com menor IDH-M, menor renda per capita, maiores taxas de enfermeiros, alta cobertura de $\operatorname{ESF}(>80 \%)$ e tinham de 0 a 0,5 unidade de saúde por mil habitantes. O Norte e o Nordeste foram as macrorregiões com mais municípios no estágio implantado (> 60\% das UBS com o e-SUS AB) em novembro de 2014, respectivamente, $38,1 \%$ e $38,4 \%$ do total de municípios (Tabela 1). As maiores taxas de procedimentos e consultas registradas no SIA/ SUS (Tabela 2) para os implantados foram nas cidades de menor porte, com cobertura de $100 \%$ de saúde da família e com maior número de unidades de saúde ( $>1$ por mil habitantes).

A análise foi realizada em dois grupos de códigos: procedimentos e consultas. Em períodos considerados como antes (junho de 2011 a maio de 2013), durante (junho de 2013 a maio de 2015) e após (junho de 2015 a maio de 2016) a implantação do sistema e-SUS AB. No grupo procedimentos (Tabela 3), a taxa média de registros no SIASUS a cada mil habitantes foi de 212,20 (DP $\pm 273,00$ ) antes, 240,60 (DP $\pm 338,90$ ) durante a implantação do sistema e de 210,60 (DP $\pm 271,70$ ) após seu uso para todos os municípios brasileiros. Já no grupo de consultas (Tabela 3), nos mesmos períodos citados, constatou-se taxas de 262,60 (DP $\pm 294,50)$ no período antes, 265,10 ( $\mathrm{DP} \pm 324,10)$ registros durante a implantação e 234,90 (DP $\pm 282,82$ ) após. Não houve diferença nas taxas de procedimentos (KruskalWallis $\mathrm{p}=0,68$ ) e consultas (Kruskal-Wallis $\mathrm{p}=0,97$ ) entre municípios com diferentes situações de implantação no período intermediário, bem como não houve aumento comparando as taxas nacionais antes e depois para procedimentos (Wilcoxon $\mathrm{p}<0,01$ [com taxas menores depois da implantação]) e consultas (Wilcoxon $p=0,14$ ). No entanto, observamos que tanto no grupo de consultas como no de procedimentos houve um aumento do registro de atendimentos entre os períodos inicial e intermediário, reduzindo novamente no período após implantação.

Para a outra análise foi realizada uma comparação entre municípios com e-SUS AB implantado (mais de 60\% das unidades utilizando o sistema $-\mathrm{n}=1.777$ ), em processo intermediário de implantação (30\% a 60\% dos serviços enviando dados $-\mathrm{n}=810$ ) e não iniciado ou incipiente (nenhuma ou ao menos uma unidade de saúde iniciou a implantação do e-SUS AB $-\mathrm{n}=2.975$ ). Para isso, foi escolhido um ponto central no período de implantação no mês de novembro de 2014 e calculada a taxa média dos meses de outubro, novembro e dezembro de 2014.

Nas análises por regressão múltipla (Tabela 4), evidenciamos que houve menores taxas de procedimentos em todas as regiões quando comparadas com a Região Norte, tendo a Nordeste uma razão de taxas (RT) de 0,34 (IC95\%: 0,30-0,40) vez menor e a Região Sul, RT = 0,35 (IC95\%: 0,30-0,41) menor do que a Região Norte. Já nas consultas, todas as regiões apresentaram taxas maiores do que Região Norte, com a Região Centro-oeste registrando RT = 1,46 (IC95\%: 1,26-1,69) mais consultas do que as demais regiões.

Os municípios mais urbanizados (> 75\%) não apresentaram diferenças nas taxas de procedimentos, porém, observou-se menores taxas de consultas em comparação a municípios com menores percentuais de urbanização (RT = 0,87; IC95\%: 0,80-0,95). Quanto ao porte, cidades com mais de 100 mil habitantes mostraram um menor registro de procedimentos (RT = 0,68; IC95\%: 0,57-0,81) e de consultas (RT = 0,54; IC95\%: 0,47-0,63) do que cidades com menos de 10 mil habitantes.

Considerando os profissionais que atuam na Atenção Básica, observa-se que uma maior taxa de médicos ( $>2$ médicos por mil habitantes) está associada ao maior número de procedimentos realizados ( $\mathrm{RT}=1,24$; IC95\%: 1,09-1,42), porém, não está associada a taxas de consultas ( $\mathrm{RT}=1,02$; IC95\%: 0,91-1,15). Quando há uma distribuição de 0,5 a 1,0 enfermeiro por mil habitantes, observa-se mais procedimentos ( $\mathrm{RT}=1,16$; IC95\%: 1,08-1,25) e consultas (RT = 1,17; IC95\%: 1,10-1,24).

Quanto aos serviços de atenção básica, nota-se que onde há mais de uma unidade de saúde por mil habitantes o registro de procedimentos é maior ( $\mathrm{RT}=1,33$; IC95\%: 1,14-1,55), mas não há diferença no número de consultas (RT = 0,96; IC95\%: 0,84-1,09). Nos municípios com cobertura de 100\% pela ESF há maior número de registros de procedimentos (RT = 1,44; IC95\%: 1,29-1,62) e de consultas (RT = 1,26; IC95\%: 1,15-1,39). Todavia, esse aumento não foi linear em relação ao aumento da cobertura. 
Tabela 1

Situação de implantação do e-SUS AB em novembro de 2014 nos municípios brasileiros, por variáveis sociodemográficas e características de serviços de saúde.

\begin{tabular}{|c|c|c|c|c|c|c|}
\hline & \multirow{2}{*}{$\begin{array}{c}\text { Incipiente } \\
\%\end{array}$} & \multirow{2}{*}{$\begin{array}{c}\text { Intermediário } \\
\%\end{array}$} & \multirow{2}{*}{$\begin{array}{c}\text { Implantado } \\
\%\end{array}$} & \multicolumn{2}{|c|}{ Total } & \multirow[t]{2}{*}{ Valor de $p$} \\
\hline & & & & $\%$ & $\mathbf{n}$ & \\
\hline Total & 53,5 & 14,5 & 32,0 & 100,0 & 5.540 & \\
\hline \multicolumn{7}{|l|}{ IDH-M } \\
\hline$<0,60$ & 44,6 & 16,7 & 38,8 & 100,0 & 1.396 & $<0,01$ \\
\hline $0,60-0,70$ & 53,5 & 15,1 & 31,5 & 100,0 & 2.263 & \\
\hline$>0,70$ & 60,1 & 12,4 & 27,5 & 100,0 & 1.903 & \\
\hline \multicolumn{7}{|c|}{ PIB per capita (Reais) } \\
\hline$<10 \mathrm{mil}$ & 46,7 & 16,9 & 36,4 & 100,0 & 2.390 & $<0,01$ \\
\hline $10-20 \mathrm{mil}$ & 57,8 & 12,9 & 29,3 & 100,0 & 1.895 & \\
\hline$>20 \mathrm{mil}$ & 59,8 & 12,8 & 27,5 & 100,0 & 1.277 & \\
\hline \multicolumn{7}{|c|}{ Taxa de médicos (por 1.000 habitantes/ano) } \\
\hline $0-1$ & 51,5 & 14,5 & 34,0 & 100,0 & 3.287 & $<0,01$ \\
\hline $1-2$ & 56,9 & 14,0 & 29,1 & 100,0 & 1.788 & \\
\hline$>2$ & 54,7 & 17,8 & 27,5 & 100,0 & 472 & \\
\hline \multicolumn{7}{|c|}{ Taxa de enfermeiros (por 1.000 habitantes/ano) } \\
\hline $0,0-0,5$ & 55,7 & 13,3 & 31,0 & 100,0 & 2.608 & 0,02 \\
\hline $0,5-1,0$ & 52,1 & 15,1 & 32,7 & 100,0 & 2.405 & \\
\hline$>1,0$ & 49,5 & 16,9 & 33,6 & 100,0 & 503 & \\
\hline \multicolumn{7}{|c|}{ Taxa de UBS (por 1.000 habitantes/ano) } \\
\hline $0,0-0,5$ & 54,4 & 11,0 & 34,6 & 100,0 & 3.565 & $<0,01$ \\
\hline $0,5-1,0$ & 50,8 & 20,1 & 29,0 & 100,0 & 1.698 & \\
\hline$>1,0$ & 56,6 & 26,7 & 16,7 & 100,0 & 281 & \\
\hline \multicolumn{7}{|c|}{ Porte populacional (habitantes) } \\
\hline$<10$ mil & 50,5 & 15,0 & 34,5 & 100,0 & 2.510 & $<0,01$ \\
\hline $10-50$ mil & 53,8 & 14,8 & 31,4 & 100,0 & 2.444 & \\
\hline $50-100 \mathrm{mil}$ & 62,5 & 11,7 & 25,9 & 100,0 & 325 & \\
\hline$>100 \mathrm{mil}$ & 67,5 & 11,7 & 20,9 & 100,0 & 283 & \\
\hline \multicolumn{7}{|c|}{ Cobertura do ESF (\%) } \\
\hline$<60$ & 63,5 & 12,2 & 24,3 & 100,0 & 980 & $<0,01$ \\
\hline $60-80$ & 54,9 & 14,8 & 30,3 & 100,0 & 896 & \\
\hline $80-99$ & 49,1 & 15,3 & 35,6 & 100,0 & 2.666 & \\
\hline 100 & 54,2 & 14,5 & 31,3 & 100,0 & 1.020 & \\
\hline \multicolumn{7}{|l|}{ Macrorregião } \\
\hline Norte & 49,2 & 12,7 & 38,1 & 100,0 & 449 & $<0,01$ \\
\hline Nordeste & 44,4 & 17,2 & 38,4 & 100,0 & 1.791 & \\
\hline Sudeste & 59,4 & 13,2 & 27,4 & 100,0 & 1.668 & \\
\hline Sul & 59,6 & 13,1 & 27,3 & 100,0 & 1.188 & \\
\hline Centro-oeste & 55,6 & 14,8 & 29,6 & 100,0 & 466 & \\
\hline
\end{tabular}

ESF: Estatégia Saúde da Família; IDH-M: Índice de Desenvolvimento Humano Municipal; PIB: Produto Interno Bruto; UBS: unidade básica de saúde.

Frente à utilização do e-SUS AB não foram notadas associações entre taxas de procedimentos ou consultas com a situação de implantação do sistema por cada município. Foram testadas interações entre a variável principal e as demais covariadas. Os resultados não foram significativos para a maioria dos casos, mas foram conflitantes e não interpretáveis quando significativos estatisticamente. A avaliação dos resíduos dos modelos finais mostrou que eles eram assimétricos, porém, não relacionados com os valores preditos. A remoção de variáveis não significativas ( $\mathrm{p}>0.05)$ dos modelos reduziu os valores de Bayesian Information Criterion (BIC) sem alterar os coeficientes das demais variáveis. 


\section{Tabela 2}

Média e desvio padrão (DP) dos procedimentos e consultas registrados no Sistema de Informação Ambulatorial do Sistema Único de Saúde (SIA/SUS) dos municípios brasileiros ( $n=5.433$ ) com e-SUS AB implantado em novembro de 2014.

\begin{tabular}{|c|c|c|c|c|}
\hline & $\begin{array}{c}\text { Taxas de procedimentos } \\
\text { (por } 1.000 \text { habitantes/mês) } \\
\text { Média (DP) }\end{array}$ & Valor de $p$ & $\begin{array}{c}\text { Taxas de consultas (por } \\
1.000 \text { habitantes/mês) } \\
\text { Média (DP) }\end{array}$ & $\begin{array}{c}\text { Valor de } p \\
\text { (Kruskal-Wallis) }\end{array}$ \\
\hline \multicolumn{5}{|l|}{ Situação } \\
\hline Não implantado/Incipiente & $243,0( \pm 338,2)$ & 0,96 & $268,1( \pm 336,8)$ & 0,81 \\
\hline Intermediário & $245,1( \pm 347,2)$ & & $264,7( \pm 315,8)$ & \\
\hline Implantado & $246,8( \pm 374,1)$ & & $271,5( \pm 342,1)$ & \\
\hline \multicolumn{5}{|l|}{ Macrorregiaão } \\
\hline Norte & $333,0( \pm 499,1)$ & $<0,01$ & $221,7( \pm 285,0)$ & $<0,01$ \\
\hline Nordeste & $186,7( \pm 257,7)$ & & $249,1( \pm 320,5)$ & \\
\hline Sudeste & $280,7( \pm 381,9)$ & & $293,4( \pm 352,9)$ & \\
\hline Sul & $205,7( \pm 270,0)$ & & $281,5( \pm 339,0)$ & \\
\hline Centro-oeste & $351,6( \pm 481,1)$ & & $269,0( \pm 356,4)$ & \\
\hline \multicolumn{5}{|l|}{ Porte populacional (habitantes) } \\
\hline$<10 \mathrm{mil}$ & $277,2( \pm 397,5)$ & $<0,01$ & $316,0( \pm 396,8)$ & $<0,01$ \\
\hline $10-50 \mathrm{mil}$ & $226,7( \pm 330,4)$ & & $245,6( \pm 289,5)$ & \\
\hline $50-100 \mathrm{mil}$ & $201,9( \pm 206,0)$ & & $195,0( \pm 181,2)$ & \\
\hline$>100 \mathrm{mil}$ & $158,5( \pm 116,2)$ & & $136,7( \pm 102,5)$ & \\
\hline \multicolumn{5}{|l|}{ IDH-M } \\
\hline$<0,60$ & $208,2( \pm 308,7)$ & $<0,01$ & $253,1( \pm 338,8)$ & $<0,01$ \\
\hline $0,60-0,70$ & $258,7( \pm 388,8)$ & & $271,3( \pm 349,7)$ & \\
\hline$>0,70$ & $254,3( \pm 331,5)$ & & $277,1( \pm 315,1)$ & \\
\hline \multicolumn{5}{|l|}{ PIB per capita (Reais) } \\
\hline$<10 \mathrm{mil}$ & $223,1( \pm 326,7)$ & $<0,01$ & $262,4( \pm 325,8)$ & $<0,01$ \\
\hline $10-20 \mathrm{mil}$ & $266,2( \pm 387,2)$ & & $283,4( \pm 367,6)$ & \\
\hline$>20 \mathrm{mil}$ & $252,4( \pm 337,4)$ & & $258,8( \pm 301,5)$ & \\
\hline \multicolumn{5}{|c|}{ Taxa de médicos (por 1.000 habitantes/ano) } \\
\hline $0-1$ & $226,6( \pm 333,1)$ & $<0,01$ & $251,3( \pm 314,6)$ & $<0,01$ \\
\hline $1-2$ & $254,4( \pm 363,2)$ & & $285,6( \pm 372,5)$ & \\
\hline$>2$ & $327,5( \pm 414,2)$ & & $322,9( \pm 322,1)$ & \\
\hline \multicolumn{5}{|c|}{$\begin{array}{l}\text { Taxa de enfermeiros (por } 1.000 \text { habitantes/ } \\
\text { ano) }\end{array}$} \\
\hline $0,0-0,5$ & $212,1( \pm 299,7)$ & $<0,01$ & $226,8( \pm 265,3)$ & $<0,01$ \\
\hline $0,5-1,0$ & $259,3( \pm 371,1)$ & & $293,5( \pm 379,5)$ & \\
\hline$>1,0$ & $323,4( \pm 440,8)$ & & $359,7( \pm 409,3)$ & \\
\hline \multicolumn{5}{|c|}{ Taxa de UBS (por 1.000 habitantes/ano) } \\
\hline $0,0-0,5$ & $236,9( \pm 325,6)$ & 0,05 & $250,1( \pm 304,6)$ & $<0,01$ \\
\hline $0,5-1,0$ & $256,3( \pm 397,6)$ & & $298,0( \pm 372,8)$ & \\
\hline$>1,0$ & $271,0( \pm 374,4)$ & & $312,3( \pm 402,7)$ & \\
\hline \multicolumn{5}{|l|}{ Cobertura do ESF (\%) } \\
\hline$<60$ & $224,6( \pm 330,6)$ & $<0,01$ & $215,4( \pm 301,4)$ & $<0,01$ \\
\hline $60-80$ & $254,3( \pm 388,8)$ & & $253,8( \pm 334,0)$ & \\
\hline $80-99$ & $242,9( \pm 346,9)$ & & $273,0( \pm 321,9)$ & \\
\hline 100 & $259,3( \pm 346,8)$ & & $322,1( \pm 390,5)$ & \\
\hline
\end{tabular}

ESF: Estatégia Saúde da Família; IDH: Índice de Desenvolvimento Humano Municipal; PIB: Produto Interno Bruto; UBS: unidade básica de saúde. Nota: não implantado/incipiente: 0-30\% das UBS com implantação; intermediário: 31-60\% das UBS com implantação e; implantado: > 60\% das UBS implantadas. 
Tabela 3

Taxas médias e desvios padrão (DP) de procedimentos e consultas por mil habitantes/mês segundo a situação de implantação do e-SUS AB em novembro de 2014

\begin{tabular}{|c|c|c|c|c|c|}
\hline & $\begin{array}{c}\text { T1 } \\
\text { Taxa média (DP) }\end{array}$ & $\begin{array}{c}\text { T2 } \\
\text { Taxa média (DP) }\end{array}$ & $\begin{array}{c}\text { T3 } \\
\text { Taxa média (DP) }\end{array}$ & Frequência & $\begin{array}{l}\text { Comparação T1-T3 } \\
\text { Valor de } p \text { (Wilcoxon) }\end{array}$ \\
\hline \multicolumn{6}{|l|}{ Taxa de procedimentos } \\
\hline Total Brasil & $212,2( \pm 273,0)$ & $240,6( \pm 338,9)$ & $210,6( \pm 271,7)$ & 5.500 & 0,14 \\
\hline Não implantado/Incipiente & $212,9( \pm 270,9)$ & $236,7( \pm 313,7)$ & $209,7( \pm 261,9)$ & 2.941 & \\
\hline Intermediário & $207,6( \pm 261,1)$ & $243,2( \pm 344,9)$ & $209,6( \pm 276,8)$ & 802 & \\
\hline Implantado & $213,3( \pm 281,8)$ & $246,0( \pm 374,7)$ & $212,5( \pm 285,3)$ & 1.757 & \\
\hline Valor de p (Kruskal-Wallis) & 0,99 & 0,97 & 0,70 & & \\
\hline \multicolumn{6}{|l|}{ Taxa de consultas } \\
\hline Total Brasil & $262,6( \pm 294,5)$ & $265,1( \pm 324,1)$ & $234,9( \pm 282,8)$ & 5.491 & $<0,01$ \\
\hline Não implantado/Incipiente & $248,4( \pm 270,5)$ & $266,3( \pm 331,1)$ & $231,1( \pm 274,4)$ & 2.944 & \\
\hline Intermediário & $260,9( \pm 275,3)$ & $258,4( \pm 289,2)$ & $244,6( \pm 298,6)$ & 797 & \\
\hline Implantado & $287,3( \pm 337,3)$ & $266,1( \pm 327,4)$ & $237,1( \pm 289,1)$ & 1.750 & \\
\hline Valor de p (Kruskal-Wallis) & $<0,01$ & 0,68 & 0,58 & & \\
\hline
\end{tabular}

Nota: não implantado/incipiente: 0-30\% das UBS com implantação; intermediário: 31-60\% das UBS com implantação e; implantado: > 60\% das UBS implantadas; T1: junho de 2011 a maio de 2013; T2: junho de 2013 a maio de 2015; T3: junho de 2015 a maio de 2016.

\section{Discussão}

O presente estudo mostra que a implantação do e-SUS AB não está associada a alterações das taxas de procedimentos e consultas notificadas no SIA/SUS. Outro achado importante foi constatar que os municípios com maior cobertura de ESF apresentaram maior registro de procedimentos e consultas em relação aos de menor cobertura. Além disso, municípios de grande porte ou com mais de $100 \mathrm{mil}$ habitantes parecem estar relacionados a um menor registro de procedimentos e consultas.

No final de 2014, aproximadamente metade dos municípios brasileiros já havia implantado o e-SUS AB em mais de $60 \%$ das suas unidades de saúde. Os municípios de pequeno porte e com maiores coberturas de ESF foram os primeiros a iniciar a utilização do e-SUS AB, já que estavam em estágio implantado no momento intermediário. Entretanto, tais municípios não apresentaram alterações nas taxas de procedimentos ou consultas notificadas em comparação a antes e depois. Teoricamente, a implantação do PEC tornaria o processo de notificação mais fácil, uma vez que o profissional tem acesso individual a um computador com internet no seu local de trabalho ${ }^{14}$, acessando o prontuário durante o atendimento, e o software produz o registro de alguns procedimentos conforme os protocolos clínicos 15 . Assim, poderia esperar-se uma redução de eventual subnotificação de alguns procedimentos. Não existe informação sobre a magnitude de subnotificações ou supernotificações, de modo que não seria previsível um aumento ou redução. Todavia, o uso da CDS mantém semelhança com o sistema anterior, exceto que os procedimentos são notificados de forma individual, constando o número do Cartão Nacional de Saúde (CNS) 14. A ausência de mudança nas notificações poderia estar relacionada a uma qualificação dos registros para uma exposição da quantidade real de procedimentos realizados, considerando que o sistema, por ter registro de atendimentos individualizados por usuário e o número do seu CNS, minimiza possíveis fraudes ou duplicações. Dadas as características de implantação, espera-se que as tecnologias de informação, como o e-SUS AB, propiciem uma melhor eficiência, custo-efetividade e segurança na prestação dos cuidados em saúde 4 .

O modelo proposto por Donabedian 16 para avaliação de serviços de saúde é baseado em informações de estrutura, processo e resultados. A dimensão estrutura poderia envolver a análise da implantação de tecnologia de avaliação como o e-SUS (PEC e CDS) e seus condicionantes; já indicadores de processo podem identificar facilidades ou dificuldades de uso que impactem no 


\section{Tabela 4}

Razões de taxas ajustadas por regressão de procedimentos e de consultas registradas no Sistema de Informação Ambulatorial do Sistema Único de Saúde (SIA/SUS) entre outubro e dezembro de 2014, segundo a situação de implantação do e-SUS AB e outras características sociodemográficas nos municípios brasileiros.

\begin{tabular}{|c|c|c|}
\hline & $\begin{array}{c}\text { Procedimentos } \\
\text { Razão de taxas * (IC95\%) }\end{array}$ & $\begin{array}{c}\text { Consultas } \\
\text { Razão de taxas * (IC95\%) }\end{array}$ \\
\hline \multicolumn{3}{|l|}{ Situação } \\
\hline Não iniciado/Incipiente & 1,00 & 1,00 \\
\hline Intermediário & $1,03(0,94-1,12)$ & $0,96(0,89-1,04)$ \\
\hline Implantado & $0,91(0,85-0,97)$ & $0,98(0,92-1,04)$ \\
\hline \multicolumn{3}{|l|}{ Macrorregião } \\
\hline Norte & 1,00 & 1,00 \\
\hline Nordeste & $0,34(0,30-0,40)$ & $1,08(0,96-1,22)$ \\
\hline Sudeste & $0,49(0,42-0,57)$ & $1,40(1,23-1,61)$ \\
\hline Sul & $0,35(0,30-0,41)$ & $1,27(1,10-1,46)$ \\
\hline Centro-oeste & $0,69(0,58-0,81)$ & $1,46(1,26-1,69)$ \\
\hline \multicolumn{3}{|c|}{ Porte populacional (habitantes) } \\
\hline$<10$ mil & 1,00 & 1,00 \\
\hline $10-50 \mathrm{mil}$ & $1,08(1,00-1,17)$ & $0,86(0,81-0,92)$ \\
\hline $50-100 \mathrm{mil}$ & $0,88(0,75-1,02)$ & $0,85(0,74-0,97)$ \\
\hline$>100 \mathrm{mil}$ & $0,68(0,57-0,81)$ & $0,54(0,47-0,63)$ \\
\hline \multicolumn{3}{|l|}{ IDH-M } \\
\hline$<0,60$ & 1,00 & 1,00 \\
\hline $0,60-0,70$ & $0,88(0,80-0,97)$ & $0,91(0,83-0,98)$ \\
\hline$>0,70$ & $0,91(0,80-1,03)$ & $0,96(0,87-1,07)$ \\
\hline \multicolumn{3}{|l|}{ PIB per capita (Reais) } \\
\hline$>10 \mathrm{mil}$ & 1,00 & 1,00 \\
\hline $10-20 \mathrm{mil}$ & $1,02(0,93-1,13)$ & $1,04(0,96-1,13)$ \\
\hline$>20 \mathrm{mil}$ & $1,03(0,91-1,16)$ & $1,00(0,91-1,10)$ \\
\hline \multicolumn{3}{|c|}{ Taxa de médicos (por 1.000 habitantes/ano) } \\
\hline $0-1$ & 1,00 & 1,00 \\
\hline $1-2$ & $1,09(1,01-1,18)$ & $1,04(0,98-1,11)$ \\
\hline$>2$ & $1,24(1,09-1,42)$ & $1,02(0,91-1,15)$ \\
\hline \multicolumn{3}{|c|}{ Taxa de enfermeiros (por 1.000 habitantes/ano) } \\
\hline $0,0-0,5$ & 1,00 & 1,00 \\
\hline $0,5-1,0$ & $1,16(1,08-1,25)$ & $1,17(1,10-1,24)$ \\
\hline$>1,0$ & $1,16(1,02-1,32)$ & $1,16(1,04-1,30)$ \\
\hline \multicolumn{3}{|c|}{ Taxa de UBS (por 1.000 habitantes/ano) } \\
\hline $0,0-0,5$ & 1,00 & 1,00 \\
\hline $0,5-1,0$ & $1,05(0,97-1,13)$ & $1,06(1,00-1,13)$ \\
\hline$>1,0$ & $1,33(1,14-1,55)$ & $0,96(0,84-1,09)$ \\
\hline \multicolumn{3}{|l|}{ Cobertura do ESF (\%) } \\
\hline$<60$ & 1,00 & 1,00 \\
\hline $60-80$ & $0,97(0,87-1,08)$ & $1,00(0,91-1,10)$ \\
\hline $80-99$ & $1,05(0,96-1,16)$ & $1,13(1,04-1,22)$ \\
\hline 100 & $1,44(1,29-1,62)$ & $1,26(1,15-1,39)$ \\
\hline
\end{tabular}

ESF: Estatégia Saúde da Família; IC95\%: intervalo de 95\% de confiança; IDH: Índice de Desenvolvimento Humano Municipal; PIB: Produto Interno Bruto; UBS: unidade básica de saúde.

Nota: não implantado/incipiente: 0-30\% das UBS com implantação; intermediário: 31-60\% das UBS com implantação e; implantado: > 60\% das UBS implantadas.

* Todas as variáveis da tabela presentes na regressão multivariável também controladas por: \% população (planos médicos), \% população (idosos > 70 anos), \% população (crianças < 10 anos), \% população (mulheres) e \% população (residente em zona urbana). 
quantitativo de notificações (como no presente estudo), mas principalmente a qualidade dos dados gerados. Considerando os resultados deste estudo, pode-se pensar que é pouco provável que a implantação do e-SUS AB, por si só, produziria alguma mudança no quantitativo de notificações por aumento do acesso, sendo mais provável uma eventual mudança de processos de trabalho e notificações, qualificando a informação. A implantação de diferentes indicadores propostos por Donabedian 16 (p.ex.: estrutura, processo, resultado) permite uma visão mais ampla da situação e aprimoramento dos serviços e de todos os profissionais envolvidos no processo.

Neste estudo, os municípios com cobertura de saúde da família em 100\% de sua população apresentaram maior notificação de procedimentos e consultas que os municípios com cobertura menor. Esses resultados poderiam estar associados ao fato de maiores coberturas de ESF serem encontradas em municípios de menor porte ou áreas periféricas de grandes centros 17, e isto poderia influenciar na utilização e acessibilidade dos serviços 18. Entretanto, as estimativas obtidas foram ajustadas por número de médicos, enfermeiras e porte municipal. Assim, uma explicação alternativa é que a saúde da família, frente ao modelo de atenção básica tradicional, apresenta resultados positivos na ampliação do acesso, cobertura de serviços e desempenho 10,17,19 devido ao trabalho em equipe multidisciplinar e à orientação de cuidado longitudinal com enfoque familiar. Além disso, outros estudos corroboram que a ESF gera um aumento de atendimentos, predominantemente de imunizações, procedimentos básicos de enfermagem, consultas médicas, visitas domiciliares 19 e procedimentos odontológicos 20 .

Outro achado relevante foi que municípios de grande porte, com mais de 100 mil habitantes, apresentaram uma taxa de procedimentos e consultas menores do que os demais. Esses municípios costumam ter maiores investimentos em serviços de média e alta complexidades 21,22 e menores gastos com atenção primária em saúde 23 . Há também diferenças entre as regiões brasileiras, e paradoxalmente a Região Norte que registra as maiores taxas de consulta, também apresenta as menores taxas de procedimentos. Outros estudos também demonstram heterogeneidades no registro de procedimentos ambulatoriais entre as regiões 24 , com taxas de procedimentos mais baixas na Região Norte 25 .

O estudo apresenta como ponto forte a inclusão de todos os municípios brasileiros, eliminando-se a possibilidade de viés de seleção. Buscamos controlar por um conjunto grande de variáveis que poderiam confundir a principal associação de interesse para dar mais validade ao resultado. Ainda assim, pode haver confundimento residual. $\mathrm{O}$ uso de dados de sistemas oficiais de informação tem implicações para a validade dos resultados. De certa forma, as informações são de procedência avalizada, porém, desconsidera-se as características específicas de registro de cada serviço ou profissional que podem gerar impactos nas notificações, assim como instabilidades na Internet ou equipamentos de informática, as quais são difíceis de mensurar. O importante é o município seguir alimentando o SIA/ SUS com regularidade, seja por intermédio do PEC, CDS ou outros softwares já anteriormente utilizados. Embora a intervenção escolhida (uso do e-SUS AB) não ser randomizada, ela é uma intervenção natural e uma oportunidade única de avaliação de uma política de abrangência em nível nacional.

Ao conhecimento dos autores, este é o primeiro estudo a avaliar algum impacto do sistema e-SUS $\mathrm{AB}$ com abrangência nacional. As conclusões não evidenciaram um aumento do registro de procedimentos ou consultas. Entretanto, outras análises quanto ao uso do sistema e as suas implicações nos processos de trabalho das equipes devem ser aprofundadas a fim de trazer diferentes dimensões desse processo a outros pesquisadores, usuários e gestores dos serviços de saúde. Foi possível traçar um perfil dos municípios que primeiro implantaram o uso do e-SUS AB, e os resultados podem apoiar os gestores na tomada de decisão sobre a utilização ou continuidade do uso do sistema, visto que o efeito não representou redução drástica de notificações. 


\section{Colaboradores}

M. A. Thum participou da concepção do estudo, coleta, interpretação dos dados, redação do manuscrito e aprovação da versão final. J. Baldisserotto colaborou na concepção do estudo, interpretação dos resultados, revisão crítica e aprovação da versão final. R. K. Celeste participou da concepção do estudo, análise dos dados, redação, interpretação dos resultados e aprovação da versão final.

\section{Informações adicionais}

ORCID: Moara Ailane Thum (0000-0003-21916619); Julio Baldisserotto (0000-0003-3211-024X); Roger Keller Celeste (0000-0002-2468-6655).

\section{Agradecimentos}

R. K. Celeste possui bolsa de produtividade do Conselho Nacional de Desenvolvimento Científico e Tecnológico (PQ2-CNPq).

\section{Referências}

1. Conselho Nacional de Secretários de Saúde. Estratégia e-SUS na Atenção Básica e Sistema de Informação em Saúde da Atenção Básica SISAB. Brasília: Conselho Nacional de Secretários de Saúde; 2013. (Nota Técnica 07/2013).

2. Oliveira AEC, Lima IMB, Nascimento JA, Coelho HFC, Santos SR. Implantação do e-SUS $\mathrm{AB}$ no Distrito Sanitário IV de João Pessoa PB: relato de experiência. Saúde Debate 2016; 40:212-8.

3. Jha AK, Ferris TG, Donelan K, DesRoches C, Shields A, Rosenbaum S, et al. How common are electronic health records in the United States? A summary of the evidence. Health Aff (Millwood) 2006; 25:w496-507.

4. Marin HF. Sistemas de informação em saúde: considerações gerais. J Health Inform 2010; 2:20-4.

5. Shekelle PG, Morton SC, Keeler EB. Costs and benefits of health information technology. Evid Rep Technol Assess (Full Rep) 2006; (132):1-71.

6. Price M, Singer A, Kim J. Adopting electronic medical records: are they just electronic paper records? Can Fam Physician 2013; 59:e322-9.

7. Ludwick DA, Doucette J. Adopting electronic medical records in primary care: Lessons learned from health information systems implementation experience in seven countries. Int J Med Inform 2009; 78:22-31.

8. Ministério da Saúde. Nota metodológica da certificação das equipes de atenção básica participantes do Programa de Melhoria do Acesso e da Qualidade na Atenção Básica. Brasília: Ministério da Saúde; 2013.

9. Santos AF, Fonseca Sobrinho D, Araujo LL, Procópio CSD, Lopes EAS, Lima AMLD, et al. Incorporação de Tecnologias de Informação e Comunicação e qualidade na atenção básica em saúde no Brasil. Cad Saúde Pública 2017; 33:e00172815.

10. Lima RTS, Fernandes TG, Balieiro AAS, Costa FS, Schramm JMA, Schweickardt JC, et al. A Atenção Básica no Brasil e o Programa Mais Médicos: uma análise de indicadores de produção. Ciênc Saúde Coletiva 2016; 21:268596.

11. Silva BP, Stockmann D, Lúcio DS, Henna E, Rocha MCP, Junqueira FM, et al. Ampliação do acesso à saúde na região mais vulnerável do Estado de São Paulo, Brasil: reflexo do Programa Mais Médicos? Ciênc Saúde Coletiva 2016; 21:2899-906.

12. Galavote HS, Zandonade E, Garcia ACP, Freitas PSS, Seidl H, Contarato PC, et al. The nurse's work in primary health care. Esc Anna Nery Rev Enferm 2016; 20:90-8.

13. Ministério da Saúde. Portaria no 1.976, de 12 de setembro de 2014. Altera e acrescenta dispositivos à Portaria no 1.412/GM/MS, de 10 de julho de 2013. Diário Oficial da União 2014; 15 set. 
14. Departamento de Atenção Básica, Ministério da Saúde. Manual de uso do sistema com Prontuário Eletrônico do Cidadão PEC v.1.3. http://dab.saude.gov.br/portaldab/esus/ma nual_pec_1.3/index.php (acessado em 18/ Out/2018).

15. Archer N, Fevrier-Thomas U, Lokker C, McKibbon KA, Straus SE. Personal health records: a scoping review. J Am Med Informatics Assoc 2011; 18:515-22.

16. Donabedian A. The quality of care. How can it be assessed? JAMA 1988; 260:1743-8.

17. Arantes LJ, Shimizu HE, Merchán-Hamann E. The benefits and challenges of the Family Health Strategy in Brazilian Primary Health care: a literature review. Ciênc Saúde Coletiva 2016; 21:1499-510.

18. Travassos C, Martins M. Uma revisão sobre os conceitos de acesso e utilização de serviços de saúde. Cad Saúde Pública 2004; 20 Suppl 2:S190-8.

19. Facchini L, Piccini R, Tomasi E, Thumé E. Desempenho do PSF no Sul e no Nordeste do Brasil: avaliação institucional e epidemiológica da atenção básica à saúde. Ciênc Saúde Coletiva $2006 ; 11: 669-81$.

20. Corrêa GT, Celeste RK. Associação entre a cobertura de equipes de saúde bucal na saúde da família e o aumento na produção ambulatorial dos municípios brasileiros, 1999 e 2011. Cad Saúde Pública 2015; 31:2588-98.

21. Cardoso MO, Vieira-da-Silva LM. Evaluation of primary care coverage in Salvador, Bahia State, Brazil (2000 to 2007). Cad Saúde Pública 2012; 28:1273-84.
22. Viana ALd'A, Rocha JSY, Elias PE, Ibañez N Novaes MHD. Modelos de atenção básica nos grandes municípios paulistas: efetividade, eficácia, sustentabilidade e governabilidade. Ciênc Saúde Coletiva 2006; 11:577-606.

23. Cabreira FS, Ritter F, Aguiar VR, Celeste RK. Despesas municipais em Atenção Primária à Saúde no Rio Grande do Sul: um estudo ecológico. Cad Saúde Pública 2018; 34:e00150117.

24. Castro AP, Neves VR, Aciole GG. Diferenças regionais e custos dos procedimentos de fisioterapia no Sistema Único de Saúde do Brasil, 1995 a 2008. Rev Panam Salud Pública 2011; 30:469-76

25. Celeste RK, Vital JF, Junger WL, Reichenheim ME. Séries de procedimentos odontológicos realizadas nos serviços públicos brasileiros, 1994-2007. Ciênc Saúde Coletiva 2011; 16:4523-32. 


\section{Abstract}

This article sought to evaluate e-SUS AB's impact on procedure and consultation notification in the Outpatient Information System of the Brazilian Unified National Health System (SIA/SUS) in Brazilian municipalities. This is an ecological study with longitudinal comparisons before, during and after the e-SUS AB implementation period (June 2013 to May 2015) and, during implementation, cross-sectional comparison between municipalities in completed, intermediary and initial implementation stages. Rates were described using means and standard deviations and analyzed through negative binomial regression. Municipalities where e-SUS $A B$ had been implemented in November 2014 were small, with lower Municipal Human Development Index (HDI-M), lower per capita income, higher rates of nurses, high Family Health Strategy - (FHS) coverage (> 80\%). We did not observe differences in rates of procedures or consultations between municipalities according to implementation stage and there was no increase over time. Municipalities with 100\% FHS coverage had higher rates of procedures (rate ratio $[R R]=$ 1.44; 95\%CI: 1.29-1.62) and consultations $(R R=$ 1.26; 95\%CI: 1.15-1.39); municipalities with more than 100 thousand inhabitants had lower rates of procedures $(R R=0.68 ; 95 \% \mathrm{CI}: 0.57-0.81)$ and consultations $(R R=0.54 ; 95 \% C I: 0.47-0.63)$. We found lower procedure rates in all regions when compared with the Northern Region, with the Northeastern RR of 0.34 (95\%CI: 0.30-0.40) lower and the Southern Region RR of 0.35 (95\% CI: 0.30-0.41) lower than the Northern Region. Further analyses of system use and its implications in teams' work processes should be carried out in order to support managers in using and maintaining the system.

Electronic Health Records; Health Information Systems; Family Health Strategy; Primary Health Care

\section{Resumen}

El objetivo fue evaluar el impacto del e-SUS $A B$ en la notificación de procedimientos y consultas, dentro del Sistema de Información Ambulatoria del Sistema Único de Salud (SIA/SUS) de los municipios brasileños. Se trata de un estudio ecológico con una comparación longitudinal antes, durante $y$ después del período de implantación del e-SUS AB (junio de 2013 a mayo de 2015). Durante la implantación, se realizó una comparación seccional entre municipios en fase implantación, intermedia e incipiente. Las tasas se describieron mediante medias y desvío estándar, analizados gracias una regresión binomial negativa. Los municipios con el e-SUS AB implantado en noviembre 2014 eran de pequeño porte, con menor Índice de Desarollo Humano Municipal (IDH-M), menor renta per capita, mayores tasas de enfermeros, alta cobertura de la Estategia de Salud Familiar ESF (> 80\%). No se observaron diferencias en las tasas de procedimientos o consultas entre municipios, de acuerdo con la fase de implantación, y no hubo aumento a lo largo del tiempo. Municipios con una cobertura del $100 \%$ de la ESF presentan mayores tasas de procedimientos (razón de tasas $[R T]=1,44$; IC95\%: 1,29-1,62) y de consultas (RT = 1,26; IC95\%: 1,15-1,39); los municipios con más de 100 mil habitantes registraron menores tasas de procedimientos $(R T=0,68$; IC95\%: 0,57-0,81) $y$ de consultas $(R T=0,54$; IC95\%: 0,47-0,63). Hubo menores tasas de procedimientos en todas las regiones, si se comparan con la Región Norte, teniendo la Región Nordeste RT de 0,34 (IC95\%: 0,30-0,40) menor y la Región Sur RT de 0,35 (IC95\%: 0,30-0,41) menor que la Región Norte. Se deben profundizar con otros análisis, en cuanto al uso del sistema y sus implicaciones en los procesos de trabajo de los equipos, para apoyar a los gestores en el uso y mantenimiento del sistema.

Registros Electrónicos de Salud; Sistemas de Información en Salud; Estrategia de Salud Familiar; Atención Primaria de Salud
Recebido em 26/Fev/2018

Versão final reapresentada em 18/Out/2018

Aprovado em 22/Out/2018 